\title{
On the Hardness of Approximating Multicut and Sparsest-Cut [Extended Abstract]
}

\author{
Shuchi Chawla* $\quad$ Robert Krauthgamer $^{\dagger} \quad$ Ravi Kumar $^{\dagger} \quad$ Yuval Rabani $^{\ddagger} \quad$ D. Sivakumar ${ }^{\dagger}$
}

\begin{abstract}
We show that the Multicut, Sparsest-Cut, and $\mathrm{MIN}-2 \mathrm{CNF} \equiv$ DELETION problems are NP-hard to approximate within every constant factor, assuming the Unique Games Conjecture of Khot [STOC, 2002]. A quantitatively stronger version of the conjecture implies inapproximability factor of $\Omega(\log \log n)$.
\end{abstract}

\section{Introduction}

In the Multicut problem the input is an undirected graph $G=(V, E)$ on $n=|V|$ vertices and $k$ pairs of vertices $\left\{s_{i}, t_{i}\right\}_{i=1}^{k}$, called demand pairs, and the goal is to find a minimum-size subset of the edges $M \subseteq E$ whose removal disconnects all the demand pairs, i.e., in the subgraph $(V, E \backslash M)$ every $s_{i}$ is disconnected from its corresponding vertex $t_{i}$. In the weighted version of this problem, the input also specifies a positive cost $c(e)$ for each edge $e \in E$ and the goal is to find a multicut $M$ whose total cost $c(M)=\sum_{e \in M} c(e)$ is minimal. This problem is known to be APX-hard [12].

We prove that if a strong version of the Unique Games Conjecture of Khot [19] is true, then MulticUT is NP-hard to approximate to within a factor of $\Omega(\log \log n)$. Under the original version of this conjecture, our reduction shows that for every constant $L>0$, it is NP-hard to approximate Multicut to within factor $L$.

*Computer Science Department, Carnegie Mellon University, Pittsburgh, PA 15213, USA. Email: shuchiecs.cmu.edu. This work is supported in part by an IBM PhD Fellowship, and by NSF grants CCR0122581 and IIS-0121678, and part of it was done while the author was visiting IBM Almaden Research Center.

${ }^{\dagger}$ IBM Almaden Research Center, 650 Harry Road, San Jose, CA 95120, USA. Email: \{robi, ravi, siva\}@almaden.ibm. com.

${ }^{\ddagger}$ Computer Science Department, Technion-Israel Institute of Technology, Haifa 32000, Israel. Part of this work was done while the author was on sabbatical leave at Cornell University, while visiting IBM Almaden Research Center, and while visiting the Institute for Pure and Applied Mathematics at UCLA. Research at the Technion supported in part by ISF grant number 52/03 and BSF grant number 02-00282. Email: rabaniecs.technion.ac.il
Our methods also yield similar bounds for SPARSESTCut and Min-2CNF $\equiv$ Deletion. The Sparsest-Cut problem has the same input as Multicut, but the goal is to find a subset of the edges $M \subseteq E$ that minimizes the ratio of $|M|$ (in the weighted version, the total cost of $M)$ to the number of demand pairs that are disconnected in $(V, E \backslash M){ }^{1}$ Since SpARSEST-CUT is not known to be APX-hard, our result gives the first indication that this problem might be hard to approximate. In the MIN-2CNF $\equiv$ DELETION problem the input is a weighted set of clauses on $n$ variables, each clause of the form $(x \equiv y)$, where $x$ and $y$ are literals, and the goal is to find a Boolean assignment to the variables minimizing the total weight of unsatisfied clauses. ${ }^{2}$ Our results also extend to the CORRELATION Clustering problem [7, 10, 13, 14] of minimizing disagreements in a weighted graph, because the approximability of this problem is known to be equivalent to that of MuLTICUT in weighted graphs [10, 14].

\subsection{Known results on Multicut, SPARSEST-Cut, and MIN-2CNF $\equiv$ DELETION}

Multicut and SpARSEST-CUT are fundamental combinatorial problems, with connections to multicommodity flow, edge expansion, and metric embeddings. Both problems can be approximated to within an $O(\log k)$ factor through linear programming relaxations [25, 16, 6, 26]. These bounds match the lower bounds on the integrality gaps up to constant factors [25, 16]. MIN-2CNF $\equiv$ DELETION can also be approximated to within an $O(\log n)$ factor, as implied by the results of Klein et al. [23], who give an approximation-preserving reduction from this problem to Multicut. Recently, starting with the groundbreaking $O(\sqrt{\log n})$-approximation for the uniform demands case [4], improved approximation algorithms have

\footnotetext{
${ }^{1}$ In general, the demand pairs may have positive weights (demands), but for our purpose of inapproximability results, it clearly suffices to consider the more restricted definition above. Our hardness results for SPARSESTCUT do not apply to the special case of uniform-demand, in which every pair of vertices forms a demand pair.

${ }^{2}$ Note that the constraints in MIN-2CNF$\equiv$ DELETION are restricted to equality (and effectively non-equality) constraints.
} 
been developed for the SPARSEST-CUT problem using a semidefinite programming relaxation [4, 11, 2]. The best approximation factor currently known for general demands is $O(\sqrt{\log k} \log \log k)$ [2]. The obvious modification of the semidefinite program used for SPARSEST-CUT to solve MulticuT was recently shown to have an integrality ratio of $\Omega(\log k)$ [1], which matches, up to constant factors, the approximation factor and integrality gap of previously analyzed linear programming relaxations for this problem.

On the hardness side, it is known that Multicut is APX-hard [12], i.e., there exists a constant $c>1$, such that it is NP-hard to approximate MULTICUT to within a factor smaller than $c$. It should be noted that this hardness of approximation holds even for $k=3$, and that the value of $c$ is not specified therein, but it is certainly much smaller than 2 . The MIN-2CNF $\equiv$ DELETION problem is also known to be APX-hard, as follows, e.g., from linear equations modulo 2 [17].

Assuming the Unique Games Conjecture, Khot [19, Theorem 3] essentially obtained an arbitrarily large constantfactor hardness for MIN-2CNF $\equiv$ DELETION, and this implies, using the aforementioned reduction of [23], a similar hardness factor for MULTICUT. These results are not noted in [19], and are weaker than our results in several respects. First, our quantitative bounds are better; thus if a stronger, yet almost as plausible, version of this conjecture is true, then our lower bound on the approximation factor improves to $L=\Omega(\log \log n)$, compared with the roughly $\Omega\left((\log \log n)^{1 / 4}\right)$ hardness that can be inferred from [19]; this can be viewed as progress towards proving tight inapproximability results for MULTICUT. Second, by qualitatively strengthening our MULTICUT result to a bicriteria version of the problem, we extend our hardness results to the SPARSEST-CUT problem. It is unclear whether Khot's reduction similarly leads to a hardness result for SPARSESTCUT. Finally, our proof is simpler (both the reduction and its analysis), and makes direct connections to cuts (in a hypercube), and thus may prove useful in further investigation of such questions.

For SPARSEST-CUT, no hardness of approximation result was previously known. Independent of our work, Khot and Vishnoi [22] have recently used a different construction to show an arbitrarily large constant factor hardness for SPARSEST-CUT assuming the Unique Games Conjecture; their hardness factor could, in principle, be pushed to $(\log \log n)^{c}$, for some constant $c>0$, assuming a stronger quantitative version of the conjecture. Additionally, they prove an integrality ratio lower bound of $\Omega\left((\log \log n)^{c}\right)$, for some fixed $c>0$, for the semidefinite program relaxations used in the recent approximation algorithms for SPARSEST-CUT.

\subsection{The Unique Games Conjecture}

Unique 2-prover game is the following problem. The input is a bipartite graph $G_{Q}=\left(Q, E_{Q}\right)$, where each side $p=1,2$ contains $n=|Q| / 2$ vertices denoted $q_{1}^{p}, \cdots, q_{n}^{p}$, and represents $n$ possible questions to prover $p$. In addition, the input contains for each edge $\left(q_{i}^{1}, q_{j}^{2}\right) \in E_{Q}$ a nonnegative weight $w\left(q_{i}^{1}, q_{j}^{2}\right)$. These edges will be called question edges, to distinguish them from edges in the MULTICUT instance. Each question to a prover is associated with a set of $d$ distinct answers, denoted by $[d]=\{1, \ldots, d\}$. The input also contains, for every edge $\left(q_{i}^{1}, q_{j}^{2}\right) \in E_{Q}$, a bijection $b_{i j}:[d] \rightarrow[d]$, which maps every answer of question $q_{i}^{1}$ to a distinct answer for $q_{j}^{2}$.

A solution $A$ to the 2-prover game consists of an answer $A_{i}^{p} \in[d]$ for each question $q_{i}^{p}$ (i.e., a sequence $\left\{A_{i}^{p}\right\}$ over all $p \in[2]$ and $i \in[n]$ ). The solution is said to satisfy an edge $\left(q_{i}^{1}, q_{j}^{2}\right) \in E_{Q}$ if the answers $A_{i}^{1}$ and $A_{j}^{2}$ agree, i.e., $A_{j}^{2}=b_{i j}\left(A_{i}^{1}\right)$. We assume that the total weight of all the edges in $E_{Q}$ is 1 (by normalization). The value of a solution is the total weight of all the edges satisfied by the solution. The value of the game is the maximum value achievable by any solution to the game.

Conjecture 1.1 (Unique Games [19]). For every fixed $\eta, \delta>0$ there exists $d=d(\eta, \delta)$ such that it is NP-hard to determine whether a unique 2-prover game with answer set size $d$ has value at least $(1-\eta)$ or at most $\delta$.

We will also consider stronger versions of the Unique Games Conjecture in which $\eta, \delta$, and $d$ are functions of $n$. Specifically, we will consider versions with $\max \{\eta, \delta\} \leq$ $1 /(\log n)^{\Omega(1)}$ and $d=d(\eta, \delta) \leq O(\log n)$. We denote the size of an input instance by $N$. Notice that $N=\left(n 2^{d}\right)^{\Theta(1)}$, and is thus polynomial in $n$ as long as $d \leq O(\log n)$, and in particular for fixed $d$.

Plausibility of the conjecture and its stronger version. The Unique Games Conjecture has been used to show optimal inapproximability results for VERTEX COVER [21] and MaX-Cut [20, 27]. Proving the conjecture using current techniques appears quite hard. In particular, the asserted NP-hardness is much stronger than what we can obtain via standard constructions using the PCP theorem [5, 3] and the parallel repetition theorem [28], two deep results in computational complexity.

Although the conjecture seems difficult to prove in general, some special cases are well-understood. In particular, if at all the Unique Games Conjecture is true, then necessarily $d \geq \max \left\{1 / \eta^{1 / 10}, 1 / \delta\right\}$. This follows from a semidefinite programming algorithm presented in [19]. Our $\Omega(\log \log n)$ hardness result (see Corollary 1.4 below) requires the existence of a constant $c>0$, such that $\max \{\eta, \delta\} \leq 1 /(\log n)^{c}$ and $d \leq O(\log n)$, which is not 
excluded by the above. Feige and Reichman [15] recently showed that for every constant $L>0$ there exists a constant $\delta>0$, such that it is NP-hard to distinguish whether a unique 2-prover game (with $d=d(L, \delta)$ ) has value at least $L \delta$ or at most $\delta$; this result falls short of the Unique Games Conjecture in that $L \delta$ is bounded away from 1 .

\subsection{Our results}

We prove the following hardness of approximation for Multicut, Sparsest-Cut, and Min-2CNF $\equiv$ DeleTION based on the Unique Games Conjecture.

Theorem 1.2. Suppose that for $\eta=\eta(n), \delta=\delta(n)$, and $d=d(\eta, \delta) \leq O(\log n)$, it is NP-hard to determine whether a unique 2-prover game with $|Q|=2 n$ vertices and answer set size $d$ has value at least $1-\eta(n)$ or at most $\delta(n)$. Then there exists $L(n)=\Omega\left(\log \frac{1}{\eta\left(n^{\Omega(1)}\right)+\delta\left(n^{\Omega(1)}\right)}\right)$ such that it is $N P$-hard to approximate MULTICUT, SPARSEST-CUT, and MIN-2CNF$\equiv$ DELETION to within factor $L(n)$.

This theorem immediately implies the following two specific hardness results.

Corollary 1.3. The Unique Games Conjecture implies that, for every constant $L>0$, it is NP-hard to approximate Multicut, Sparsest-Cut, and Min-2CNF $\equiv$ DeleTION to within factor $L$.

Corollary 1.4. The stronger version of the Unique Games Conjecture in which $\max \{\eta, \delta\} \leq 1 /(\log n)^{\Omega(1)}$, and $d=$ $d(\eta, \delta) \leq O(\log n)$, implies that for some fixed $c>0$, it is NP-hard to approximate Multicut, SPARSEST-CUT, and MIN-2CNF $\equiv$ DELETION to within factor $c \log \log n$.

For SPARSEST-CUT our hardness results hold only for the search version (in which the algorithm needs to produce a cutset and not only its value), since our proof employs a Cook reduction.

\subsection{Preliminaries}

Regular Unique Games. A unique 2-prover game is called regular if the total weight of question edges incident at any single vertex is the same, i.e., $1 / n$, for every vertex in $Q$. We now show that we can assume without loss of generality that the graph in the Unique Games Conjecture is regular. For simplicity, we state this only for fixed $\eta$ and $\delta$. A similar result holds when they depend on $n$, because we increase the input size by no more than a polynomial factor, and increase $\eta$ and $\delta$ by no more than a constant factor.

Lemma 1.5. The Unique Games Conjecture implies that for every fixed $\eta, \delta>0$, there exists $d=d(\eta, \delta)$ such that it is NP-hard to decide if a regular unique 2-prover game has value at least $1-\eta$ or at most $\delta$.
The proof is given in Appendix A and is based on an argument of Khot and Regev [21, Lemma 3.3]. The dependence of $d$ on $\eta$ and $\delta$ is important for our purposes. We thus point out that this argument does not change $d=d(\eta, \delta)$, and increases the size of the instance by at most a polynomial factor in $n$. This is acceptable in the setting of Theorem 1.2 , since the requirement $d=d(\eta, \delta) \leq O(\log n)$ is maintained and only the unspecified constants therein are affected.

Bicriteria Multicut. Our proof for the hardness of approximating SPARSEST-CUT relies on a generalization of Multicut, where the solution $M$ is required to cut only a certain fraction of the demand pairs. For a given graph $G=(V, E)$, a subset of the edges $M \subseteq E$ will be called a cutset of the graph. A cutset whose removal disconnects all the demand pairs is a multicut.

An algorithm is called an $(\alpha, \beta)$-bicriteria approximation for Multicut if, for every input instance, the algorithm outputs a cutset $M$ that disconnects at least $\alpha$ fraction of the demands and has cost at most $\beta$ times that of the optimum multicut. In other words, if $M^{*}$ is the least cost cutset that disconnects all the $k$ demand pairs, then $M$ disconnects at least $\alpha k$ demand pairs and $c(M) \leq \beta \cdot c\left(M^{*}\right)$.

Hypercubes, dimension cuts, and antipodal vertices. As usual, the $d$-dimensional hypercube (in short a $d$-cube) is the graph $C=\left(V_{C}, E_{C}\right)$ with the vertex set $V_{C}=\{0,1\}^{d}$, and an edge $(u, v) \in E_{C}$ for every two vertices $u, v \in$ $\{0,1\}^{d}$ that differ in exactly one dimension (coordinate). An edge $(u, v)$ is called a dimension-a edge, for $a \in[d]$, if $u$ and $v$ differ in dimension $a$, i.e., $u \oplus v=1_{a}$ where $1_{a}$ is a unit vector along dimension $a$. The set of all the dimension$a$ edges in the hypercube is called the dimension- $a$ cut in the hypercube; a dimension cut is a dimension- $a$ cut for some dimension $a$. The antipode of a vertex $u$ is the (unique) vertex $\bar{u}$ all of whose coordinates are different from those of $u$, i.e., $\bar{u}=u \oplus \mathbf{1}$ where $\mathbf{1}$ is the vector with 1 in every coordinate. Notice that $v$ is the antipode of $u$ if and only if $u$ is the antipode of $v$; thus, $\langle u, \bar{u}\rangle$ form an antipodal pair. The following simple fact will be key in our proof.

Fact 1.6. In any hypercube, a single dimension cut disconnects every antipodal pair.

Organization. In Section 2 we prove the part of Theorem 1.2 regarding the MULTICUT problem; our proof will actually hold for bicriteria approximation for MulticuT. We will then show in Section 3 that this stronger result yields a similar hardness of approximation for SPARSEST-CUT. Finally, in Section 4, we modify the reduction to obtain a hardness of approximation for MIN-2CNF $\equiv$ DELETION. 


\section{Hardness of bicriteria approximation for Multicut}

In this section we prove the part of Theorem 1.2 regarding the Multicut problem, namely, that the Unique Games Conjecture implies that it is NP-hard to approximate MULTICUT within a certain factor $L$. Our proof will actually show a stronger result-for every $\alpha \geq 7 / 8$ it is NP-hard to distinguish between whether there is a multicut of cost less than $n 2^{d+1}$ (the YES instance) or whether every cutset that disconnects at least $\alpha k$ demand pairs has cost at least $n 2^{d+1} L$ (the NO instance). This implies that it is NP-hard to obtain an $(\alpha, L)$-bicriteria approximation for Multicut.

We start by describing a reduction from unique 2-prover game to MULTICUT (Section 2.1), and then proceed to analyze the YES instance (Section 2.2) and the NO instance (Sections 2.3 and 2.4). Finally, we discuss the gap that is created for a bicriteria approximation of MULTICUT (Section 2.5 ).

\subsection{The reduction}

Given a unique 2-prover game instance $G_{Q}=\left(Q, E_{Q}\right)$ with $n=|Q| / 2$ and the corresponding edge weights $w(e)$ and bijections $b_{i j}:[d] \rightarrow[d]$, we construct a MULTICUT instance $G=(V, E)$ with demand pairs, as follows. For every vertex (i.e., question) $q_{i}^{p} \in Q$, construct a $d$-dimensional hypercube $C_{j}^{p}$; the dimensions in this cube correspond to answers for the question $q_{j}^{p}$. . For each of the $2 n$ hypercubes, we let the edges inside the hypercube have cost 1 , and call them hypercube edges.

For each question edge $\left(q_{i}^{1}, q_{j}^{2}\right) \in E_{Q}$, we extend $b_{i j}$ (in the obvious way) to a bijection from the vertices of $C_{i}^{1}$ to the vertices of $C_{j}^{2}$, and denote the resulting bijection by $b_{i j}^{\prime}:\{0,1\}^{d} \rightarrow\{0,1\}^{d}$. Formally, for every $u \in\{0,1\}^{d}$ (vertex in $C_{i}^{1}$ ) and every $a \in[d]$, the $a$-th coordinate of $b_{i j}^{\prime}(u)$ is given by $\left(b_{i j}^{\prime}(u)\right)_{a}=u_{b_{i j}^{-1}(a)}$. Then, we connect every vertex $u \in C_{i}^{1}$ to the corresponding vertex $b_{i j}^{\prime}(u) \in$ $C_{j}^{2}$ using an edge of cost $w_{i j} \Lambda$, where $\Lambda=n / \eta$ is a scaling factor. These edges are called cross edges.

Denote the resulting graph by $G=(V, E)$. Notice that $V$ is simply the union of the vertex sets of the hypercubes $C_{i}^{p}$, for all $p \in[2]$ and $i \in[n]$, and that the edge set $E$ contains two types of edges, hypercube edges and cross edges.

To complete the reduction, it remains to define the demand pairs. For a vertex $u \in V$, the antipode of $u$ in $G$, denoted $\bar{u}$, is defined to be the antipodal vertex of $u$ in the hypercube $C_{i}^{p}$ that contains $u$. The set $D$ of demand pairs then

\footnotetext{
${ }^{3}$ This is a standard technique in PCP constructions for graph optimization problems. A hypercube can be interpreted as a "long code" [8], and a dimension cut is the encoding of an answer in the 2-prover game.
}

contains every pair of antipodal vertices in $G$, and hence $k=|D|=n 2^{d-1}$. Note that every vertex of $G$ belong to exactly one demand pair.

\subsection{The YES instance}

Lemma 2.1. If there is a solution A for the unique 2-prover game $G_{Q}$ such that the total weight of the satisfied questions is at least $1-\eta$, then there exists a multicut $M \subseteq E$ for the Multicut instance $G$ such that $c(M) \leq 2^{d+1} n$.

Proof. Let $A$ be such a solution for $G_{Q}$. Construct $M$ by taking the following edges. For every question $q_{i}^{p} \in Q$ and the corresponding answer $A_{i}^{p}$ (of prover $p$ ), take the dimension- $A_{i}^{p}$ cut in cube $C_{i}^{p}$. In addition, for every edge $\left(q_{i}^{1}, q_{j}^{2}\right) \in E_{Q}$ that the solution $A$ does not satisfy, take all the cross edges between the corresponding cubes $C_{i}^{1}$ and $C_{j}^{2}$.

We first claim that removing $M$ from $G$ disconnects all the demand pairs. To see this, we define a Boolean function $f: V \rightarrow\{0,1\}$ on the graph vertices. For every cube $C_{i}^{p}$, consider the dimension- $A_{i}^{p}$ cut; it disconnects the cube into two connected components, one containing the all zeros vector $\mathbf{0}$ and one containing the all ones vector $\mathbf{1}$. For every $v \in C_{i}^{p}$, let $f(v)=0$ if $v$ is in the same side as $\mathbf{0}$, and $f(v)=1$ otherwise. This is exactly the $A_{i}^{p}$-th bit in $v$, i.e., $f(v)=v_{A_{i}^{p}}$. Now consider any demand pair $(v, \bar{v})$, and note that $f(v)=1-f(\bar{v})$. We will show below that every edge $(u, v) \notin M$ satisfies the property $f(u)=f(v)$. This clearly proves the claim.

Consider first a hypercube edge $(u, v)$ in $C_{i}^{p}$ that is not a dimension- $A_{i}^{p}$ edge. Then $f(u)=u_{A_{i}^{p}}=v_{A_{i}^{p}}=f(v)$, by the definition of $f$. Next consider a cross edge $(u, v) \notin M$. Then this edge lies between cubes $C_{i}^{1}$ and $C_{j}^{2}$, such that the question edge $\left(q_{i}^{1}, q_{j}^{2}\right)$ satisfied by the unique 2-prover game solution $A$. Therefore, $b_{i j}\left(A_{i}^{1}\right)=A_{j}^{2}$. Then, $f(u)=$ $u_{A_{i}^{1}}=v_{b_{i j}\left(A_{i}^{1}\right)}=v_{A_{j}^{2}}=f(v)$.

Finally, we bound the cost of the solution. Let $S$ be the set of question edges not satisfied by the solution $A$. The total cost of the multicut solution is thus $c(M)=2 n 2^{d-1}+$ $2^{d} \Lambda \sum_{\left(Q_{i}^{1}, Q_{j}^{2}\right) \in S} w_{i j} \leq 2^{d} n+2^{d} \frac{n}{\eta} \eta=2^{d+1} n$.

\subsection{Hypercube cuts and influences}

We will analyze the NO instance shortly, but first we set up some notation and present a few technical lemmas regarding cuts in hypercubes. In particular, we present Lemma 2.3, which will have a crucial role in that analysis.

Recall that the dimensions of the hypercubes in the multicut instance correspond to answers to the 2-prover game. Therefore, we define the extent to which a dimension participates in a cut on the cube as follows. Let $C=\left(V_{C}, E_{C}\right)$ be a $d$-dimensional hypercube. For a function $f: V_{C} \rightarrow \mathbb{R}$, 
the influence of dimension $a \in[d]$ (a.k.a. the influence of the a-th variable) on the function, denoted $I_{a}^{f}$, is defined to be the fraction of dimension- $a$ edges $(u, v) \in E_{C}$ for which $f(u) \neq f(v)$. For a cutset $M \subseteq E_{C}$, the influence of dimension $a \in[d]$ on the cutset, denoted $I_{a}^{M}$, is defined as the fraction of dimension- $a$ edges that belong to $M$. Observe that $|M|=2^{d-1} \sum_{a \in[d]} I_{a}^{M}$.

Proposition 2.2. Let $M \subseteq E_{C}$ be a cutset in a hypercube $C=\left(V_{C}, E_{C}\right)$. Define $g: V_{C} \rightarrow \mathbb{Z}$ by labeling the connected components of $C \backslash M$ by distinct integers, and letting $g(v)$ for $v \in V_{C}$ be the label of the connected component containing $v$. Then $I_{a}^{M} \geq I_{a}^{g}$.

Proof. Observe that the cutset $M$ must contain every edge $(u, v) \in E_{C}$ for which $g(u) \neq g(v)$.

The lemma below shows that if a cutset $M$ has few edges (i.e., small cost) but its removal disconnects a large fraction of the antipodal pairs in the hypercube $C$, then there must be a dimension $a \in[d]$ with large influence.

Lemma 2.3. Let $M$ be a cutset in a d-dimensional hypercube $C$, and suppose that removing $M$ disconnects at least $\beta$ fraction of the antipodal pairs in $C$. Then for all $x>0$,

$$
\sum_{a \in[d]} I_{a}^{M} \leq \beta x \Rightarrow \max _{a \in[d]} I_{a}^{M} \geq 2^{-6 x} / 27 .
$$

To prove this, we will make use of the following lemma, due to Kahn, Kalai, and Linial [18] (see also [29. Section 1.5]).

Lemma 2.4 (Kahn, Kalai, and Linial [18]). Let $f$ be a Boolean function defined on a hypercube, and suppose the fraction of inputs $x$ for which $f(x)=1$ is $p \leq 1 / 2$. Then for all $\alpha>0$,

$$
\frac{1}{\alpha} \sum_{i} I_{i}^{f}+\sum_{i}\left(I_{i}^{f}\right)^{4 / 3} \geq 2 p \frac{\log \alpha}{\alpha} .
$$

We note that the proof of Lemma 2.4 is based on Fourier analysis of Boolean functions, and that its statement above follows from the proofs therein.

Proof of Lemma 2.3. We first convert the cutset $M$ into a two-sided (binary) cut. Observe that each connected component of $C \backslash M$ must have size at most $2^{d}-\beta 2^{d-1}=$ $(1-\beta / 2)\left|V_{C}\right|$. If there is a component of size larger than $\left|V_{C}\right| / 2$, we combine the rest of the components into a single component. Otherwise, we split the set of components into two parts such that the total size of the components in each part is at most $\frac{2}{3}\left|V_{C}\right|$. Call the resulting cutset $M^{\prime}$. Note that $M^{\prime} \subseteq M$ and thus, for every $a \in[d]$, the influence of every dimension in $M^{\prime}$ is no larger than its counterpart in $M$, i.e., $I_{a}^{M^{\prime}} \leq I_{a}^{M}$. Hence, $\sum_{a} I_{a}^{M^{\prime}} \leq \sum_{a} I_{a}^{M} \leq \beta x$. This twosided cut defines a Boolean function $f: V_{C} \rightarrow\{0,1\}$ with balance $p \leq 1 / 2$ satisfying $p \geq \min \{\beta / 2,1 / 3\} \geq \beta / 3$ and $I^{M_{a}^{\prime}}=I_{i}^{f}$. Using Lemma 2.4 with $\alpha=2^{2 x}$, we have

$$
\frac{\beta x}{2^{2 x}}+\sum_{a \in[d]}\left(I_{a}^{M^{\prime}}\right)^{4 / 3} \geq 2 \frac{\beta}{3} \frac{2 x}{2^{2 x}}
$$

We thus obtain

$$
\sum_{a}\left(I_{a}^{M^{\prime}}\right)^{4 / 3} \geq \frac{\beta}{3} \frac{x}{2^{2 x}}
$$

Now set $y=\max _{a \in[d]} I_{a}^{M^{\prime}}$. Then we get

$$
\sum_{a}\left(I_{a}^{M^{\prime}}\right)^{4 / 3} \leq y^{1 / 3} \sum_{a} I_{a}^{M^{\prime}} \leq \beta x y^{1 / 3}
$$

Therefore, we have $y^{1 / 3} \geq \frac{1}{3} 2^{-2 x}$, or, $y \geq 2^{-6 x} / 27$.

The next lemma shows that if two functions $f, g: V_{C} \rightarrow$ $\mathbb{R}$ agree on most of the inputs $v \in V_{C}$, then their influences are quite similar.

Lemma 2.5. Let $C=\left(V_{C}, E_{C}\right)$ be a hypercube. If for two functions $f, g: V_{C} \rightarrow \mathbb{R}$ we have $f(v)=g(v)$ for all but $a$ $\gamma$ fraction of inputs $v \in V_{C}$, then for every dimension a we have $\left|I_{a}^{f}-I_{a}^{g}\right| \leq 2 \gamma$.

Proof. Suppose that $C$ is a $d$-dimensional hypercube, and consider a dimension- $a$ edge $(u, v) \in E_{C}$. By our assumption, for all but at most $\gamma 2^{d}$ such edges, we must have $f(u)=g(u)$ and $f(v)=g(v)$, and in particular $f(u)-f(v)=g(u)-g(v)$. Recalling that there are exactly $2^{d-1}$ dimension- $a$ edges, and that $I_{a}^{f}$ is the fraction of those edges for which $f(u)-f(v) \neq 0$ (and similarly for $g$ ), we conclude that $f(u)-f(v)=g(u)-g(v)$ for at most $2 \gamma$ fraction of the dimension- $a$ edges, and thus $\left|I_{a}^{f}-I_{a}^{g}\right| \leq 2 \gamma$.

\subsection{The NO instance}

Lemma 2.6. There exists $L=\Omega(\log 1 /(\eta+\delta))$ such that if the Multicut instance $G$ has a cutset of cost at most $2^{d} n L$ whose removal disconnects $\alpha \geq 7 / 8$ fraction of the demand pairs, then there is a solution A for the unique 2prover game $G_{Q}$ whose value is larger than $\delta$.

Proof. Let $L=c \log 1 /(\eta+\delta)$ where $c>0$ is a constant to be determined later, and let $M \subseteq E$ be a cutset of cost $c(M) \leq 2^{d} n L$ whose removal disconnects $\alpha \geq 7 / 8$ fraction of the demand pairs. Using $M$, we will construct for the unique 2-prover game $G_{Q}$ a randomized solution $A$ whose expected value is larger than $\delta$, thereby proving the existence of a solution of value larger than $\delta$. Without loss of generality, we may assume that $M$ is minimal with respect to containment, namely, for every subset $M^{\prime} \subseteq M$, if $M^{\prime} \neq M$ then removing $M^{\prime}$ from $G$ disconnects fewer demand pairs than removing $M$ would. Given such a minimal 
cutset $M$, for each cube $C_{i}^{p}$ in $G$, consider the cutset $M$ induces in this cube, and let $I_{a}^{p, i}$ be the influence of dimension $a \in[d]$ on this cutset. The randomized solution $A$ (i.e., a strategy for the two provers) is defined as follows. For each vertex (question) $q_{i}^{p} \in Q$, we choose $A_{i}^{p}$ to be the answer (dimension) $a^{\prime} \in[d]$ with probability $I_{a^{\prime}}^{p, i} / \sum_{a \in[d]} I_{a}^{p, i}$.

We proceed to analyze the expected value of this randomized solution $A$. Recall that the value of a solution corresponds to the probability that, for a question edge $\left(q_{i}^{1}, q_{j}^{2}\right)$ chosen at random with probability proportional to its weight, we have $a_{j}^{2}=b_{i j}\left(a_{i}^{1}\right)$. Notice that although $q_{i}^{1}$ and $q_{j}^{2}$ are correlated, each one is uniformly distributed because $Q$ is regular. Without loss of generality, we assume removing $M$ disconnects at least as many demand pairs inside the cubes $\left\{C_{l}^{1}\right\}_{l \in[n]}$ as inside the cubes $\left\{C_{l}^{2}\right\}_{l \in[n]}$. Now we claim that with a high probability over the choice of a question edge, the cut $M$ has a low cost over edges incident on the corresponding hypercubes, and disconnects many demand pairs in the hypercubes. In other words, the quality of the cut locally is nearly as good as the quality of the cut globally. In particular, we upper bound the probability of the following four "bad" events (for a choice of a question edge $\left.\left(q_{i}^{1}, q_{j}^{2}\right)\right)$ :

$\mathcal{E}_{1}=$ fewer than half the demand pairs in $C_{i}^{1}$ are disconnected in $G \backslash M$.

$\mathcal{E}_{2}=M$ contains more than $2^{d+2} L$ hypercube edges in $C_{i}^{1}$. $\mathcal{E}_{3}=M$ contains more than $2^{d+2} L$ hypercube edges in $C_{j}^{2}$. $\mathcal{E}_{4}=M$ contains more than $2^{d} / 2^{96 L+7}$ cross edges between $C_{i}^{1}$ and $C_{j}^{2}$.

First, by our assumption above, removing $M$ disconnects at least $\alpha \geq 7 / 8$ fraction of the demand pairs inside the cubes $\left\{C_{l}^{1}\right\}_{l \in[n]}$, and thus by Markov's inequality, $\operatorname{Pr}\left[\mathcal{E}_{1}\right] \leq 1 / 4$. Next, the cutset $M$ contains at most $2^{d} n L$ hypercube edges, thus the expected number of edges in $C_{i}^{1} \cup C_{j}^{2}$ that are contained in $M$ is at most $2^{d} L$, and $\operatorname{Pr}\left[\mathcal{E}_{2} \cup \mathcal{E}_{3}\right] \leq 1 / 2$. Finally, if $c>0$ is sufficiently small, $\operatorname{Pr}\left[\mathcal{E}_{4}\right] \leq \eta L 2^{96 L+7} \leq \eta^{1 / 2} \leq$ $1 / 8$, as otherwise the total cost along the corresponding question-edges $\left(q_{i}^{1}, q_{j}^{2}\right)$ (i.e., those for which the cutset $M$ contains more than $2^{d} / 2^{96 L+7}$ cross edges between $C_{i}^{1}$ and $\left.C_{j}^{2}\right)$ is more than $\left(\eta L 2^{96 L+7}\right) \cdot\left(2^{d} / 2^{96 L+7}\right) \cdot(n / \eta)=$ $n 2^{d} L \geq c(M)$. Taking a union bound, we upper bound the probability that any of the bad events occurs by

$$
\operatorname{Pr}\left[\mathcal{E}_{1} \cup \mathcal{E}_{2} \cup \mathcal{E}_{3} \cup \mathcal{E}_{4}\right] \leq \frac{7}{8} .
$$

In order to lower bound the expected value of the randomized solution $A$, we would like to show that if none of the above bad events happens, then there exists a dimension $a^{*} \in[d]$, such that in cube $C_{i}^{1}$ this dimension $a^{*}$ has large influence, $I_{a^{*}}^{1, i}$, and in cube $C_{j}^{2}$ dimension $b_{i j}\left(a^{*}\right)$ has large influence, $I_{b_{i j}\left(a^{*}\right)}^{2, j}$. For the cube $C_{i}^{1}$, if the event $\mathcal{E}_{2}$ does not occur, then $\sum_{a \in[d]} I_{a}^{1, i} \leq 8 L$. If neither $\mathcal{E}_{1}$ nor $\mathcal{E}_{2}$ occurs, then we can use Lemma 2.3 (with $\beta=1 / 2, x=16 L$ ) and conclude that there exists a dimension $a^{*} \in[d]$ such that

$$
I_{a^{*}}^{1, i} \geq 2^{-96 L} / 27 \text {. }
$$

For the sake of analysis, label the connected components of $G \backslash M$ with distinct integer values. Define $f: C_{i}^{1} \rightarrow \mathbb{Z}$ by letting $f(v)$ for $u \in C_{i}^{1}$ be the label of the connected component of $u$, and define $g: C_{j}^{2} \rightarrow \mathbb{Z}$ similarly. For every $u \in C_{i}^{1}$, if $f(u) \neq g\left(b_{i j}^{\prime}(u)\right)$ then the cross edge $\left(u, b_{i j}^{\prime}(u)\right)$ must be contained in the cutset $M$, and because we assumed the event $\mathcal{E}_{4}$ happens, this occurs for at most $2^{d} / 2^{96 L+7}$ vertices $u \in C_{i}^{1}$. Furthermore, by the definition of $f$ and $g$, we have $I_{a}^{1, i}=I_{a}^{f}$ and $I_{a}^{2, j}=I_{a}^{g}$. Applying Lemma 2.5 to the functions $f$ and $g \circ b_{i j}^{\prime}$, we conclude that $\left|I_{a}^{f}-I_{a}^{g \circ b_{i j}^{\prime}}\right| \leq 2^{-96 L-6}$ for all dimensions $a \in[d]$. Finally, since $b_{i j}$ is just a permutation of the coordinates, for all we have $a \in[d], I_{a}^{g \circ b_{i j}^{\prime}}=I_{b_{i j}(a)}^{g}$. Altogether, we obtain

$$
I_{b_{i j}\left(a^{*}\right)}^{2, j} \geq I_{a^{*}}^{1, i}-2^{-96 L-6} \geq 2^{-96 L} / 54,
$$

and thus

$$
\begin{aligned}
\operatorname{Pr}\left[A_{j}^{2}=b_{i j}\left(A_{i}^{1}\right)\right] & \geq \operatorname{Pr}\left[A_{i}^{1}=a^{*}, A_{j}^{2}=b_{i j}\left(a^{*}\right)\right] \\
& \geq \frac{1}{8} \cdot \frac{I_{a^{*}}^{1, i}}{\sum_{a \in[d]} I_{a}^{1, i}} \cdot \frac{I_{a^{*}}^{2, j}}{\sum_{a \in[d]} I_{b_{i j}(a)}^{2, j}} \\
& \geq \Omega\left(L^{-2} 2^{-96 L}\right)
\end{aligned}
$$

We conclude that the expected value of the randomized solution $A$ is

$$
\sum_{(i, j) \in E_{Q}} w\left(q_{i}^{1}, q_{j}^{2}\right) \operatorname{Pr}\left[A_{j}^{2}=b_{i j}\left(A_{i}^{1}\right)\right] \geq \Omega\left(L^{-2} 2^{-96 L}\right)>\delta,
$$

where the last inequality holds if $c>0$ is sufficiently small, and this completes the proof of Lemma 2.6 .

\subsection{Putting it all together}

The above reduction from unique 2-prover game to Multicut produces a gap of $L(n)=\Omega(\log 1 /(\eta(n)+$ $\delta(n)))$. We assumed $d(\eta, \delta) \leq O(\log n)$, and thus the resulting Multicut instance $G$ has size $N=\left(n 2^{d}\right)^{O(1)}=$ $n^{\Theta(1)}$. It follows that in terms of the instance size $N$, the gap is $L(N)=\Omega\left(\log 1 /\left(\eta\left(N^{\Theta(1)}\right)+\delta\left(N^{\Theta(1)}\right)\right)\right)$.

This completes the proof of the part of Theorem 1.2 regarding the MULTICUT problem, namely, that the Unique Games Conjecture implies that it is NP-hard to approximate MuLTICUT within the above factor $L(N)$. In fact, the above proof shows that it is even NP-hard to obtain a $(7 / 8, L(N))$ bicriteria approximation. 
Note that the number of demand pairs is $k=n 2^{d-1}=$ $n^{\Theta(1)}$, and thus the hardness of approximation factor is similar when expressed in terms of $k$ as well. Note also that all edge weights in the MULTICUT instance constructed above are bounded by a polynomial in the size of the graph. Therefore, via a standard reduction, a similar hardness result holds for the unweighted Multicut problem as well.

\section{Hardness of approximating SPARSEST-CUT}

In this section we prove the part of Theorem 1.2 regarding the Sparsest-Cut problem. The proof follows immediately from the next lemma in conjunction with the hardness of bicriteria approximation of MULTICUT (from the previous section).

Lemma 3.1. Let $0<\alpha<1$ be a constant. If there exists a polynomial-time algorithm for SPARSEST-CUT that produces a cut whose value is within factor $\rho \geq 1$ of the minimum, then there is a polynomial time algorithm that computes an $\left(\alpha, \frac{2 \rho}{1-\alpha}\right)$-bicriteria approximation for MULTICUT.

Proof. Fix $0<\alpha<1$, and suppose $\mathcal{A}$ is a polynomial-time algorithm for SPARSEST-CUT that produces a cut whose value is within factor $\rho \geq 1$ of the minimum. Now suppose we are given an input graph $G=(V, E)$ and $k$ demand pairs $\left\{s_{i}, t_{i}\right\}_{i=1}^{k}$. We may assume without loss of generality that every $s_{i}$ is connected (in $G$ ) to its corresponding $t_{i}$. Let $c_{\min }$ and $c_{\max }$ be the smallest and largest edge costs in $G$, and let $n=|V|$.

We now describe the bicriteria approximation algorithm for Multicut. For every value $C \in\left[c_{\min }, n^{2} c_{\max }\right]$ that is a power of 2 , execute a procedure that we will describe momentarily to compute a cutset $M_{C} \subseteq E$, and report, from all these cutsets $M_{C}$ whose removal disconnects at least $\alpha k$ demand pairs, the one of least cost. For a given value $C>0$, the procedure starts with $M_{C}=\emptyset$, and then iteratively "augments" $M_{C}$ as follows: Take a connected component $S$ of $G \backslash M_{C}$, apply algorithm $\mathcal{A}$ to $G[S]$ (the subgraph induced on $S$ and all the demand pairs that lie inside $S$ ), and if the resulting cutset $E_{S}$ has value (in $G[S]$ ) at most $\frac{\rho}{1-\alpha} \cdot \frac{C}{k}$, then add the edges $E_{S}$ to $M_{C}$. Here, the value (ratio of cost to demands cut) of $E_{S}$ is defined as $b_{S}=c\left(E_{S}\right) /\left|D_{S}\right|$, where $D_{S}$ is the collection of demand pairs that lie in $G[S]$ and get disconnected (in $G[S]$ ) when $E_{S}$ is removed. Proceed with the iterations until for every connected component $S$ in $G \backslash M_{C}$ we have $b_{S}>\frac{\rho}{1-\alpha} \frac{C}{k}$, at which point the procedure returns the cutset $M_{C}$.

This algorithm clearly runs in polynomial time. To analyze its performance, we first claim that for every value $C$, the cutset $M_{C}$ returned by the above procedure has sparsestcut value (ratio of cost to demand disconnected, in $G$ ) at most $\frac{\rho}{1-\alpha} \frac{C}{k}$. Indeed, suppose the procedure performs $t$ augmentation iterations. Denote by $S_{i}$ the connected component $S$ that is cut at iteration $i \in[t]$, by $E_{S_{i}}$ the corresponding cutset output by $\mathcal{A}$, and by $D_{S_{i}}$ the corresponding set of demand pairs that get disconnected. Clearly, $M_{C}$ is the disjoint union $E_{1} \cup \cdots \cup E_{t}$, and it is easy to verify that the collection $D_{C}$ of demand pairs cut by the cutset $M_{C}$ is the disjoint union $D_{S_{1}} \cup \cdots \cup D_{S_{t}}$. Thus,

$$
\begin{aligned}
c\left(M_{C}\right)=\sum_{i=1}^{t} c\left(E_{S_{i}}\right) & \leq \frac{\rho}{1-\alpha} \cdot \frac{C}{k} \sum_{i=1}^{t}\left|D_{S_{i}}\right| \\
& =\frac{\rho}{1-\alpha} \cdot \frac{C}{k}\left|D_{C}\right|,
\end{aligned}
$$

which proves the claim.

For the sake of analysis, fix an optimal multicut $M^{*} \subseteq$ $E$, i.e., a cutset of $G$ whose removal disconnects all the demand pairs and has the least cost. The sparsest-cut value of $M^{*}$ is $b^{*}=c\left(M^{*}\right) / k$. We will show that if $C \in$ $\left[c\left(M^{*}\right), 2 c\left(M^{*}\right)\right]$, then the above procedure produces a cutset $M_{C}$ whose removal disconnects a collection $D_{C}$ containing $\left|D_{C}\right| \geq \alpha k$ demand pairs; this will complete the proof of the lemma, because it immediately follows that

$$
c\left(M_{C}\right) \leq \frac{\rho}{1-\alpha} \cdot \frac{C}{k}\left|D_{C}\right| \leq \frac{\rho}{1-\alpha} \cdot 2 c\left(M^{*}\right),
$$

and clearly $c\left(M^{*}\right) \in\left[c_{\min },\left(\begin{array}{l}n \\ 2\end{array}\right) \cdot c_{\max }\right]$. So suppose now $C \in\left[c\left(M^{*}\right), 2 c\left(M^{*}\right)\right]$ and assume for contradiction that $\left|D_{C}\right|<\alpha k$. Denote by $V_{1}, \ldots, V_{p} \subseteq V$ the connected components of $G \backslash M_{C}$, and let $D_{j}$ contain the demand pairs that lie inside $V_{j}$. It is easy to see that $\sum_{j=1}^{p}\left|D_{j}\right|=$ $k-\left|D_{C}\right|>(1-\alpha) k$. Similarly, let $M_{j}^{*}$ be the collection of edges in $M^{*}$ that lie inside $V_{j}$. Then $c\left(M^{*}\right) \geq$ $\sum_{j=1}^{p} c\left(M_{j}^{*}\right)$. Notice that, in every induced graph $G\left[V_{j}\right]$, the edges of $M_{j}^{*}$ form a cutset (of $G\left[V_{j}\right]$ ) that cuts all the demand pairs in $D_{j}$. Using the stopping condition of the procedure, and since $\mathcal{A}$ provides an approximation within factor $\rho$, we have $c\left(M_{j}^{*}\right) \geq \frac{1}{1-\alpha} \frac{C}{k}\left|D_{j}\right|$ (the inequality is not strict because $D_{j}$ might be empty). We thus derive the contradiction

$$
c\left(M^{*}\right) \geq \sum_{j=1}^{p} c\left(M_{j}^{*}\right) \geq \frac{1}{1-\alpha} \cdot \frac{C}{k} \sum_{j=1}^{p}\left|D_{j}\right|>c\left(M^{*}\right) .
$$

This shows that when $C \in\left[c\left(M^{*}\right), 2 c\left(M^{*}\right)\right]$, the procedure stops with a cutset $M_{C}$ whose removal disconnects $\left|D_{C}\right| \geq$ $\alpha k$ demand pairs, and concludes the proof of the lemma.

\section{Hardness of approximating $\mathrm{MIN}-2 \mathrm{CNF} \equiv$ DELETION}

In this section, we modify the reduction in Section 2.1 to obtain a hardness of approximation for MIN-2CNF $\equiv$ 
Deletion. In particular, we reduce the Multicut instance obtained in Section 2.1 to MIN-2CNF $\equiv$ DELETION, such that a solution to the latter gives a MulticuT of the same cost in the former.

The MIN-2CNF $\equiv$ DELETION instance contains $2^{d-1} n$ variables, one for each demand pair $(u, \bar{u})$. In particular, for every demand pair $(u, \bar{u}) \in D$, we associate the literal $x_{u}$ with $u$ and the literal $x_{\bar{u}}=\neg x_{u}$ with $\bar{u}$. For every edge $e=(u, v)$ in the graph $G$ there is a clause $\left(x_{u} \equiv x_{v}\right)$ whose weight is equal to the edge-weight $w_{e}$.

The following lemma is immediate from the construction and implies an analog of Lemma 2.6 for MIN-2CNF $\equiv$ DELETION.

Lemma 4.1. Given an assignment $S$ of cost $W$ to the above instance of MIN-2CNF $\equiv$ DELETION, we can construct a solution of cost $W$ to the MULTICUT instance $G$.

Proof. Let $M$ be the set of edges $(u, v)$ for which $S\left(x_{u}\right) \neq$ $S\left(x_{v}\right)$. Then $M$ corresponds to the clauses that are not satisfied by $S$ and has weight $W$. The lemma follows from observing that $M$ is indeed a multicut $-S$ is constant over connected components in $G \backslash M$, and for any demand pair $(u, \bar{u}), S\left(x_{u}\right) \neq S\left(x_{\bar{u}}\right)$.

We now give an analog of Lemma 2.1 .

Lemma 4.2. If there is a solution A for the unique 2-prover game $G_{Q}$ such that the total weight of the satisfied questions is at least $1-\eta$, then there exists an assignment $S$ for the above MIN-2CNF $\equiv$ DELETION instance such that $c(S) \leq$ $2^{d+1} n$.

Proof. Given the solution $A$ for $G_{Q}$, we construct an assignment $S$ as follows. For every question $q_{i}^{p}$ and for every vertex $u$ in the corresponding hypercube $C_{i}^{p}$, define $S\left(x_{u}\right)$ to be the $A_{i}^{p}$-th bit of $u$, i.e., $S\left(x_{u}\right)=u_{A_{i}^{p}}$. Note that this is a valid assignment, i.e., $S\left(x_{u}\right)=1-S\left(\stackrel{i}{x}_{\bar{u}}\right)$ for all vertices $u$, as $u_{A_{i}^{p}}=1-\bar{u}_{A_{i}^{p}}$.

We bound the cost of the solution by first analyzing the clauses corresponding to hypercube edges in the corresponding MUlticut instance. Consider unsatisfied clauses containing both variables in the same hypercube $C_{i}^{p}$, and note that the hypercube edges corresponding to these clauses form a dimension- $A_{i}^{p}$ cut in the cube $C_{i}^{p}$. Therefore, the total weight of these clauses is at most $\left(2^{d-1}\right)(2 n)=$ $2^{d} n$.

Finally, consider an unsatisfied clause $\left(x_{u} \equiv x_{v}\right)$ corresponding to vertices in different hypercubes $C_{i}^{1}$ and $C_{j}^{2}$. Then $S\left(x_{u}\right) \neq S\left(x_{v}\right)$ implies that $u_{A_{i}^{1}}=v_{b_{i j}\left(A_{i}^{1}\right)} \neq v_{A_{j}^{2}}$, or, $b_{i j}\left(A_{i}^{1}\right) \neq A_{j}^{2}$. There are at most $2^{d}$ such clauses for each question pair not satisfied by the solution $A$. Therefore, the total weight of such clauses is at most $2^{d} \frac{n}{\eta} \eta=$ $2^{d} n$.

The lemma follows from adding the two costs.
Lemmas 4.1 and 4.2 along with Lemma 2.6 imply the part of Theorem 1.2 regarding MIN-2CNF $\equiv$ DELETION.

\section{Concluding remarks}

Several important questions are left open. First, one would like to eliminate the dependence on the Unique Games Conjecture, and obtain a "standard" hardness of approximation result. Yet another challenge is to improve the hardness factor. For MULTICUT, the $\Omega(\log n)$ integrality ratio lower bound of [1] suggests that the inapproximability bound may be improved. In particular, $(\log n)^{c}$ hardness for a constant $c>1 / 2$ will separate the approximability of Multicut from that of SPARSEST-CuT (in light of the recent approximation due to [2]).

The main bottleneck to improving the hardness factor lies in Lemma 2.3, which in turn crucially depends on Lemma 2.4, due to [18]. These bounds are tight in general, as shown by the tribes function [9]. However, in our context, in the reduction to the (non-bicriteria) MULTICUT problem, one may additionally assume that $f$ is odd, that is, $f(u) \neq f(\bar{u})$ for all inputs $u$ (because a multicut should separate all the antipodal demand pairs). Even with this additional assumption, our $\Omega(\log \log n)$ bound cannot be improved substantially, as demonstrated by the following variant of the tribes function [24]: Partition the variables $u_{1}, \ldots, u_{d}$ into subsets of size $\log d-2 \log \log d$ each; the output is the value of the first unanimous subset (under an arbitrary ordering), or $u_{1}$ if no unanimous tribe exists. This function is clearly odd, yet all variables have influence at most $O\left(\frac{\log ^{2} d}{d}\right)$ and the total influence is $O(\log d)$. For Lemma 2.3, this function leads to a cutset $M$ with $\beta=1$, such that for $x=\sum_{a} I_{a}^{M}=O(\log d)$ we have $\max _{a} I_{a}^{M} \leq 2^{-\Omega(x)}$.

A third challenge is to obtain hardness of approximation results for the uniform-demand case of the SPARSEST-CUT problem or for the BALANCED-CUT problem. Our results do not apply to this special but important case; in particular, if a 2-prover system has a low-cost balanced cut, then the corresponding graph on hypercubes would have a low-cost balanced cut regardless of the value of the 2-prover game. Alternatively, of course, one might improve the approximation algorithms for any of these problems.

\section{References}

[1] A. Agarwal, M. Charikar, K. Makarychev, and Y. Makarychev. $O(\sqrt{\log n})$ approximation algorithms for Min UnCut, Min 2CNF Deletion, and directed cut problems. In Proceedings of the 37th Annual ACM Symposium on Theory of Computing, 2005. 
[2] S. Arora, J. R. Lee, and A. Naor. Euclidean distortion and the sparsest cut. In Proceedings of the 37th Annual ACM Symposium on Theory of Computing, 2005.

[3] S. Arora, C. Lund, R. Motwani, M. Sudan, and M. Szegedy. Proof verification and the hardness of approximation problems. Journal of the ACM, 45(3):501-555, 1998.

[4] S. Arora, S. Rao, and U. Vazirani. Expander flows, geometric embeddings, and graph partitionings. In Proceedings of the 36th Annual ACM Symposium on Theory of Computing, pages 222-231, 2004.

[5] S. Arora and S. Safra. Probabilistic checking of proofs: A new characterization of NP. Journal of the ACM, 45(1):70$122,1998$.

[6] Y. Aumann and Y. Rabani. An $O(\log k)$ approximate mincut max-flow theorem and approximation algorithm. SIAM Journal on Computing, 27(1):291-301, 1998.

[7] N. Bansal, A. Blum, and S. Chawla. Correlation Clustering. Machine Learning, Special Issue on Clustering, 56(1-3):89113, 2004.

[8] M. Bellare, O. Goldreich, and M. Sudan. Free bits, PCP's and non-approximability - towards tight results. SIAM Journal on Computing, 27(3):804-915, 1998.

[9] M. Ben-Or and N. Linial. Collective coin flipping. In Randomness and Computation, pages 91-115, 1990.

[10] M. Charikar, V. Guruswami, and A. Wirth. Clustering with qualitative information. In Proceedings of the 44th IEEE Symposium on Foundations of Computer Science, pages 524-533, 2003

[11] S. Chawla, A. Gupta, and H. Räcke. Improved approximations to sparsest cut. In Proceedings of the 16th Annual ACM-SIAM Symposium on Discrete Algorithms, pages 102$111,2005$.

[12] E. Dahlhaus, D. S. Johnson, C. H. Papadimitriou, P. D. Seymour, and M. Yannakakis. The complexity of multiterminal cuts. SIAM Journal on Computing, 23(4):864-894, 1994.

[13] E. Demaine and N. Immorlica. Correlation clustering with partial information. In Proceedings of the 6th International Workshop on Approximation Algorithms for Combinatorial Optimization Problems (APPROX), pages 1-13, 2003.

[14] D. Emanuel and A. Fiat. Correlation clusteringminimizing disagreements on arbitrary weighted graphs. In Proceedings of 11th Annual European Symposium on Algorithms, pages 208-220, 2003.

[15] U. Feige and D. Reichman. On systems of linear equations with two variables per equation. In Proceedings of the 7th International Workshop on Approximation Algorithms for Combinatorial Optimization Problems (APPROX), pages 117-127, 2004.

[16] N. Garg, V. V. Vazirani, and M. Yannakakis. Approximate max-flow min-(multi)cut theorems and their applications. SIAM Journal on Computing, 25(2):235-251, 1996.

[17] J. Håstad. Some optimal inapproximability results. Journal of the ACM, 48(4):798-859, 2001.
[18] J. Kahn, G. Kalai, and N. Linial. The influence of variables on boolean functions. In Proceedings of the 29th IEEE Symposium on Foundations of Computer Science, pages 68-80, 1988.

[19] S. Khot. On the power of unique 2-prover 1-round games. In Proceedings of the 34th Annual ACM Symposium on Theory of Computing, pages 767-775, 2002.

[20] S. Khot, G. Kindler, E. Mossel, and R. O'Donnell. Optimal inapproximability results for MAX-CUT and other 2variable CSPs. In Proceedings of the 45th IEEE Symposium on Foundations of Computer Science, pages 146-154, 2004.

[21] S. Khot and O. Regev. Vertex cover might be hard to approximate to within 2- $\epsilon$. In Proceedings of the 18th Annual IEEE Conference on Computational Complexity, pages 379-386, 2003.

[22] S. Khot and N. Vishnoi. On embeddability of negative type metrics into $\ell_{1}$. Manuscript, 2004.

[23] P. Klein, A. Agrawal, R. Ravi, and S. Rao. Approximation through multicommodity flow. In Proceedings of the 31st IEEE Symposium on Foundations of Computer Science, pages 726-737, 1990.

[24] R. Kumar, D. Sivakumar, and E. Vee. Manuscript, 2005.

[25] F. T. Leighton and S. Rao. An approximate max-flow mincut theorem for uniform multicommodity flow problems with applications to approximation algorithms. In Proceedings of the 29th IEEE Symposium on Foundations of Computer Science, pages 422-431, 1988.

[26] N. Linial, E. London, and Y. Rabinovich. The geometry of graphs and some of its algorithmic applications. Combinatorica, 15(2):215-245, 1995.

[27] E. Mossel, R. O'Donnell, and K. Oleszkiewicz. Noise stability of functions with low influences: Invariance and optimality. Manuscript, 2005.

[28] R. Raz. A parallel repetition theorem. SIAM Journal on Computing, 27(3):763-803, 1998.

[29] D. Stefankovic. Fourier Transforms in Computer Science. $\mathrm{PhD}$ thesis, University of Chicago, 2000.

\section{A. Regularity of the Unique Games instance}

Proof of Lemma 1.5 Given a unique 2-prover game $Q$, we describe how to convert it to a regular game while preserving its completeness and soundness. First we claim that we can assume that the ratio between the maximum weight $\max _{e} w_{e}$ and the minimum weight $\min _{e} w_{e}$ is bounded by $n^{3}$. This is because we can remove all edges with weight less than $\frac{1}{n^{3}} \max _{e} w_{e}$ from the graph, changing the soundness and completeness parameters by at most $\frac{1}{n}$. By a similar argument, we can assume that all weights in the graph are integral multiples of $t=\frac{1}{n^{2}} \min _{e} w_{e}$.

Now we convert $Q$ to a regular graph $Q^{\prime}$ as follows. For each prover $p \in\{1,2\}$ and question $q_{i}^{p}$, form $W(p, i) / t$ 
vertices $q_{i}^{p}(1), \cdots, q_{i}^{p}(W(p, i) / t)$, where $W(p, i)$ is the total weight of all the edges incident on $q_{i}^{p}$. For every pair of vertices $\left(q_{i}^{1}, q_{j}^{2}\right)$, connected by an edge $e$ in $Q$, we form an edge between $q_{i}^{1}(x)$ and $q_{j}^{2}(y)$, for all possible values of $x$ and $y$, with weight $w_{e} \frac{t}{W(1, i)} \frac{t}{W(2, j)}$.

Note that the total weight of all the edges remains the same as before. Each new vertex $q_{i}^{1}(x)$ has total weight $\sum_{e} w_{e} \frac{t}{W(1, i)} \frac{t}{W(2, j)} \frac{W(2, j)}{t}=t$, where the sum is over all edges $e$ incident on $q_{i}^{1}$. Therefore, the graph is regular. Furthermore, the number of vertices increases by a factor of at most $n^{5}$.

It only remains to show that the soundness and completeness parameters are preserved. To see this, note that any solution on the original graph $Q$ can be transformed to a solution of the same value on $Q^{\prime}$, by picking the same answer for every vertex $q_{i}^{p}(x)$ in $Q^{\prime}$ as the answer picked for $q_{i}^{p}$ in $Q$. Likewise, consider a solution in $Q^{\prime}$. Note that the answers for the questions $q_{i}^{p}(x)$ with different values of $x$ must all be the same, because all these questions are connected to identical sets of vertices, with the same weights. Therefore, the solution in $Q$ that picks the same answer for $q_{i}^{p}$ as the answer for $q_{i}^{p}(x)$ in $Q^{\prime}$ has the same weight as the given solution in $Q^{\prime}$.

Thus for every solution in $Q$, there is a solution of the same weight in $Q^{\prime}$ and vice versa. This proves that the two games have exactly the same soundness and completeness parameters. 